
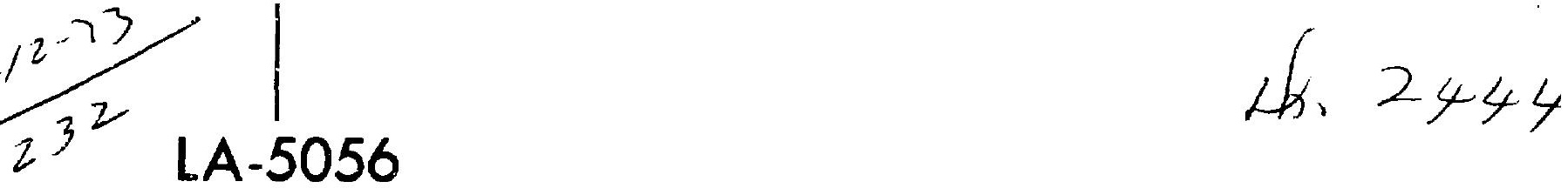

\title{
The Electromagnetic Pulse
} from an Underground Nuclear Explosion



\section{scientific laboratory}

of the University of Californic IOS ALAMOS, NEW MEXICO 87544

1

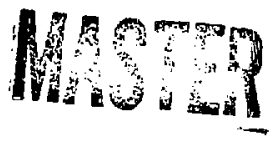


This report was prepared as an account of york sponsored by the United States Government. Neither the United Stetes nor the United States Atomic Energy Commission, nor any of their employees, nor any of their contractors, subcontractors, or their employees, makes any warranty, express or implied, or assumes any legal liability or responsibility for the accuracy, completeness or usefulness of any information, apparatus, product or process disclosed, or represents that its use wnuld not infringe privately owned rights.

Printed in the United States of America. Available from National Technical Information Service

U. S. Department of Commerce 5285 Port Royal Road

Springfield, Virginia 22151

Price: Printed Copy \$3.00; Microfiche $\$ 0.95$ 


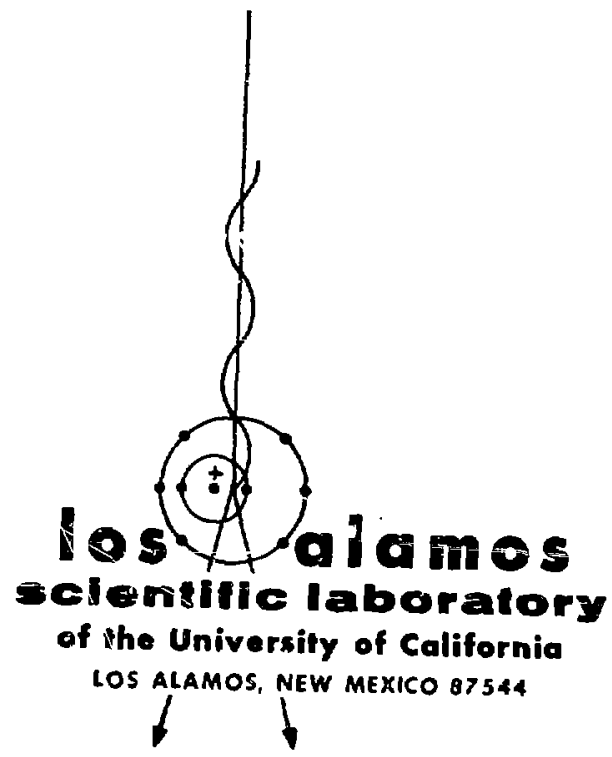

LA-5056

UC-34

ISSUED: January 1973

\title{
The Electromagnetic Pulse
}

\section{from an Underground Nuclear Explosion}

by

\author{
Leston W. Miller
}

This report was prepared as an escoum of work sponsored by the United States Government. Neither the United States nor the United States Alomic Energy Co United States nor the United states Alomic Energy Commission, nor any of their employees, nor any of their contractors, subcontractors, or their employees,

makes any wartanty, express or impljed, or essumes any

legal liability or tesponsibulity for the accuracy, com pleteness or usefulness of any information, apparñtus, product or process disclosed, or represents that its. use would not infringe privately ownes rights. 
THE ELECTROMAGNETIC PIISSE FRONI AN UNILERGROLND NUCLEAR EXPLOSION

by

Lieston W. Miller

\section{ABSTRACT}

It is argued that because of earth's high electrical conductivity, the electric current density produced by an undergrourd nuclear explosion becomes approxinately solenoidal, 1.e., free of slectric charges, in a neglisibly short time. Therefore, the bignal radiated from a simple example of a solenoidal current distribution, a polnt maginetic dipole with delta function time dependence, located underground, is calculated. The unclerground signal consists of a magnetic fleld ansociation whts a iuci weaker electric field, both of whtch picopagate through the conducting earth in a way that resembles diffusion. The aignal is reflected or trangmitted at the surface of the srouind in a way that depends upon the orlentation of the source dipole. It is shown that for a horizontal dipole, a measurable signal could be transmitted to remote poi, ts above ground.

\section{INTRODUCTION}

There are two processes that one should expect to drastically reduce the amplitude of the electromagnetic pulse (EMP) produced above the surface of the ground by an underground nuclear explosion. One of these processes 18 absorption due to the earth's electrical ccnductivity; the other is reflection at the surface of the ground due to the atrupt thange in conductivity and dielectric constant there. This report deals with the propagation of a signal from an underground source through the underground regtion and through the ground surface to remote points in the armosphere and is thus concerned malnly with these two processes. Mechanisms whereby an underground nuclear explosion could produce an EMP source are not discussed.

It is shown that the nature of the radiated slgnai is almost completely determined by the elecatcal properties of the earth, rather than by the source, if the source duration is a few microseconds or 1ess. The ceason for this is that the signal's h1gh-frequency componenta are much more strongly absorbed than its low-frequericy components. The signal propagates through the earth by a process more simllar to diffusion than to radiation. Therefore, the sigal's source 18 assumed to have a delta function time dependence. The results obtained would be the same if the source were a pulse in the microsecond range.

Furthermore, che source is assumed to be completely immersed in a cionducting medium; there are no Ingulators separating a current distribution from the surrounding earth. The medium's conductivity is assumed to be so high that any charge distributions decay in a time that is very short conpared to any time of Interest in this problem. Therefore, the underground current deneity is taken to have zero divergence, consistent with the assumption of zero charge density. Thus, it is assumed that the underground nuclear explosion produces locally a current distribution that lasts much longer than an utiderground charge distribution does, but a much shorter time than that characteriatic of an electromagnetic pulse that has propagated throigh a few hundred meters of earth.

Because the ground's conductivity tends to eliminate the high frequencles, the wavelengthis in the radiated signal are much longer then the rource 
dimensions. Therefore, the source is taken to be a delta function of position, as well as of time. The earth is treated as a uniform, isotropic, conducting medium; at the earth's surface the conductivity is assumed to go discontinuously from its uniform underground value to zero above ground. This approximation is fustifled because the wavelengths in the radiated signal are extremely long compared to the distance over which the conductivity varles significantly. Note that with this approximation the signal calculated at the ground surface just above the source is probably only qualitatively correct. The reasoin is that, as is shown below, part of the signal is reflected at the surface where the conductivity changes fron its underground value to its above ground value of zero. In actual earth this surface Is effectively somewhat below ground; the earth's electrical properties vary rapldly but not discontinuously near the ground surface. Therefore, to calculate the signal accurately within this transition region, one must probably consider the variation of conductivity with depth. Since this is not known very well, the calculation of the signal in the region near the source was done without considering surface effects, 1.e., the earth was taken to be of Infinite extent. Consequently, the results of this part of the calculation can anly be regarded as a qualitative representation of yosible experimental results.

However, in calculating the signal that propagates through the surface to remote polats in the atmosphere, both underground absocpicic ard reflection effects can be treated meaningfully by assuing uniform underground conductivity. This calculation shows that if the energy in the electromagnetic field at a time when it has propagated underground 30 m from its source is equivalent to $10^{-6} \mathrm{kt}$ of $\mathrm{TNT}$, the EMP should be observable above ground a few kilometers from ground zero for a shot at a depth of $300 \mathrm{~m}$. The signal from a source with delta function time dependeace would consist of a pulse in the 0.1 msec range followed by a slow tall of much smaller amplitude. Far from ground zero, the EMP would be a vertically polarized puls: propagating in the earth-1onosphere waveguide.

\section{THE UNDERGROUND FIELD}

Maxwell's equations in a medium of conductivity $\sigma$ and dielectric constant $\varepsilon$ are

$$
\begin{aligned}
& \nabla \times \vec{E}+\frac{1}{c} \vec{B}=0, \quad \nabla \cdot \vec{B}=0, \\
& \nabla \times \vec{B}-\frac{E}{c} \vec{E}=\frac{4 \pi}{c}\left(\sigma \vec{E}+\vec{J}_{0}\right), \quad \nabla \cdot \vec{E}=4 \pi \rho .
\end{aligned}
$$

The source current density is $\vec{J}_{0}$; since it is assumed to be solenoidal, 1.e., $\nabla \cdot J_{0}=0$, it can be expressed as the curl of a vector. Thus,

$$
\vec{J}_{0}=c \nabla \times \vec{M}(\vec{r}, t)
$$

and if the magnetic field $\vec{B}$ is written as

$$
\vec{B}-\vec{H}+4 \pi \vec{M}(\vec{r}, t),
$$

Maxwe: 1 's equations become

$$
\begin{aligned}
& \nabla \times \vec{E}+\frac{1}{c} \overrightarrow{\dot{H}}=-\frac{4 \pi}{c} \overrightarrow{\dot{M}}, \\
& \nabla \times \vec{H}-\frac{\varepsilon \vec{E}}{c}-\frac{4 \pi \sigma}{c} \vec{E}=0 .
\end{aligned}
$$

The introduction of the vector $\vec{M}$ does not imply that the medium is magnetic. It is merely a mathematically conventent vay of expressing the source current. $\vec{M}$ is taken so be

$$
\vec{M}(\vec{r}, t)=\vec{m} \delta(\vec{r}) \delta(t) ;
$$

1.e., the source is assumed to be a point magnetic dipole of moment $\vec{m} \delta(t)$ at the origin of the coordinate system. The field ti identical to the source region.

Equations (1) and (2) are most conveniently solved by Introducing a Hertz vector $H$, in terms of which

$$
\begin{aligned}
& \vec{E}=-\frac{1}{c} \frac{\partial}{\partial t} \nabla \times \vec{I}, \\
& \vec{H}=\nabla \cdot \vec{I}-\frac{1}{c} \frac{\partial}{\partial t}\left(\frac{\varepsilon}{c} \frac{\partial}{\partial t}+\frac{4 \pi \sigma}{c}\right) \vec{H},
\end{aligned}
$$

where the Cartesian components of It Batisfy

$$
-\nabla^{2} \vec{I}+\frac{\varepsilon}{c^{2}} \overrightarrow{\frac{a}{I}}+\frac{4 \pi \sigma}{c^{2}} \vec{I}=4 \pi \vec{H}(\vec{r}, t) \text {. }
$$


At this point it is ugeful to introduce a quanc1ty

$$
q=\frac{c}{4 \pi \sigma},
$$

which has the units of length. The ground conductivity is taken to be $\sigma \simeq 10^{-2}$ mho/m or, In Gaussian units, $\sigma \simeq 9 \times 10^{7} \mathrm{sec}^{-1}$, so $q \simeq 30 \mathrm{~cm}$. With this substitution for $\sigma$ and the above expression substituted for $\vec{M}(\vec{r}, t)$, the equation for $\vec{I}$ is

$$
-\nabla^{2} \vec{\Pi}+\frac{\varepsilon}{c^{2}} \overrightarrow{\vec{n}}+\frac{1}{q c} \vec{f}=4 \pi \vec{m} \delta(\vec{r}) \delta(t) .
$$

From the spherically symetrical nature of the sourse, it is clear that for each component of the vector $t$ there 18 a particular solution that is spherically symmetrical. Thus, a particular sol.tion for $I^{t}$ is

$$
\vec{\hbar}_{0}=\frac{\vec{m}}{2 \pi} \int_{-\infty}^{\infty} d \omega e^{-1 \omega t} \frac{e^{1 \Gamma r}}{r},
$$

where

$$
\Gamma^{2}=\frac{i \omega}{q c}+\frac{\omega^{2}}{c_{c}^{2}} .
$$

It is easily verifled that this is a silution by substituting it in Eq. (6) and noting that

$$
\left(-\nabla^{2}-\Gamma^{2}\right) \frac{e^{1 \Gamma r}}{r}=4 \pi \delta(\vec{r})
$$

and

$$
\frac{1}{2 \pi} \int_{-\infty}^{\infty} d \omega e^{-i \omega t}=\delta(t)
$$

For Eq. (7) to riepresent a solution that propagates outward from the source and $1 \mathrm{~s}$ attenuated as It goes; the real part of $I$ must have the same sign as $\omega$ and the imaginary part must be positive for elther sign of $\omega$. From 1ts definition 1 t $1 \mathrm{~s}$ clear that there is a square root of $\Gamma^{2}$ that satisfies these requirements for elther aign of $\omega$; hereat ter $\Gamma$ will be taken to mean that square root. With $\Gamma$ so defined, it is clear that if $\Gamma$ is replaced by $-\Gamma$ In Eq. (7) the result is again a solution for $t$, but it does not represent an outwezd-propagating signal and therefore must be rejected. If $\sigma=0$ or $q=\infty$, the solution given by Eq. (7) becomes $(\mathrm{m} / \mathrm{r}) \delta(t-\sqrt{\varepsilon} \mathrm{r} / \mathrm{c})$, as can be seen by comparing
Eq. (10). Th1s represents a thin shell propagating outward at velocity $c / \sqrt{E}$ and decreasing in amplitude as $1 / \tau$.

For the present problem, the term $1 \omega /$ qc in $\Gamma^{2}$ is dowinant. This can be seen most clearly if $\Gamma$ is witten as $1 / \mathrm{q}$ times a furction of $\mathrm{wq} / \mathrm{c}$. Thus,

$$
e^{1 \Gamma r}=\exp \left[1(r / q) \sqrt{1 \omega q / c+\varepsilon(\omega q / c)^{2}}\right] .
$$

Clearly, If $r \gg q$ then $e^{1 \Gamma r}$ is very small unless $\underline{\omega}_{c} q \ll 1$, and this implies that the term in $\Gamma$ that is proportional. to $E$ is negligible. Thus, the frequencies that make the main contribution to $\Pi_{0}$ in Eq. (7) for $r \gg q$ are those for which displacement currents, which are proportional to $E$, are negltglile compared to conduction currenta, which are proportional to $1 / q$. Thereiore, in the underground region for $r$ many times greater than $q$,

$$
\vec{I}_{0} \simeq \frac{\vec{m}}{2 \pi} \int_{-\infty}^{\infty} d \omega e^{-1 \omega t} \frac{e^{1 r} \sqrt{1 \omega / q c}}{r} .
$$

To evaluate this integral, it is desirable to distort the Integration path Into the complex $w$ plane. In so dotng, care must be exerclsed to see that the signs of the real and Imaginary parts of $\sqrt{i w / q c}$ remaln the proper ones discussed above. This can be done by setting

$$
\sqrt{\frac{1 \omega}{q c}}=u e^{1 \frac{\psi}{2}} \text {, }
$$

or

$$
i \omega=q c u^{2} e^{1 \psi},
$$

where $\psi 18$ an angle between 0 and $2 \pi$, and $u 1 s$ real and positive. For real, positive $\omega$ the value of $\psi$ is $\psi_{+}=\pi / 2$, and for real, negative $a$ the value of $\psi$ 1s $\psi_{-}=3 \pi / 2$. For an arbitrayy point in the complex $\omega$ plane, $\psi$ is the angle measured counterclockwise between the negative Imaginary axis and a straight line from the origin to the point in question. Because of the branch point at $\omega=0$ in the Integrand of Eq. (12), a branch cut is taken from the origin along the negative imaglnery axis to $-1 \infty$. The Integration path can now be distorted by choosing different values of $\psi_{+}$and $\psi_{-}$between 0 and $2 \pi$, and the signs of the real and imagtnary parts of $\sqrt{1 \omega / q c}$ w11 remaln those that correspond 
to a solution that propagates outward from the source.

For positive values of $t$, the integration path in $\mathrm{Eq}$. (12) can be closed in the negative imaginary half-plane of $\omega$, except for a circult around the branch cut. Only the branch-cut part contribites; consequently the integration path can be distorted to that shown In FIg. I. The path runs from -10 to zero along the left-hand side of the branch cut and then back to -10 along the right-hand side. On the left alde $\psi=2 \pi$, so $e^{1 r} \sqrt{\text { Iw/qc }}=e^{\text {Iur }}$, while on the right side $\psi=0$, so $\mathrm{e}^{\text {Ir } \sqrt{\text { Iw/qc }}}=\mathrm{e}^{+1 u r}$. Thus, If tine integration varlable in Eq. (12) Is changed to $u, 1 . e ., d w=-21 q c u d u$, then that equation becomes

$\tilde{I}_{0}=\frac{\overrightarrow{\underline{m}}}{2 \pi r}(-21 q c)\left(\int_{\infty}^{\bullet} u d u e^{-q c t u^{2}} e^{-1 u r}\right.$

$$
\begin{gathered}
\left.\quad+\int_{0}^{\infty} u d u e^{-q c t u^{2}} e^{+i u r}\right) \\
-\frac{-1 \vec{m} q c}{\pi r} \int_{-\infty}^{\infty} u d u e^{-q c t u^{2}}+1 u r
\end{gathered}
$$

If the sưure of the exponent is completed, the result is

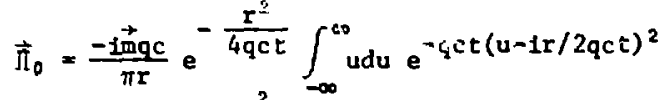

$$
\begin{aligned}
& =\frac{-\overrightarrow{\lim q c}}{\pi r} e^{-\frac{r^{2}}{4 q c t}} \int_{-\infty}^{\infty} d x\left(x+\frac{1 r}{2 q c t}\right) e^{-q c t x^{2}} \\
& =\frac{\vec{m}}{2 \pi t} e^{-\frac{r^{2}}{4 q c t}} \sqrt{\frac{\pi}{q c t}} \\
& =4 \pi q c+\frac{+}{m}(4 \pi q c t)^{-3 / 2} e^{-r^{2} / 4 q c t} \text {. }
\end{aligned}
$$

where $x$ is substituted for $u-1 r / 2 q c t$, and $1 t$ is noted that in the resulting integral only the part of the lategrand that is even in $x$ contributes.

Note that Ito as a function of $r$ is a Gaussian function whose width increases with $\sqrt{t}$. This 18 characteristic of the solutions of problems of heat conduction or diffustor where the source is a delta function of position and time as discussed by Sommerfeld. 1 Equation (6) with $\varepsilon$ set equal to zero Is a diffusion equation. It is well known that the magnetic field in a conducting medium at rest behaves In a way that can be described as diffusion. (For example, see Cowling. ${ }^{2}$ ) Thus, it is not surprising that the radiative solution that is obtained In the case of zero conductivity is so drastica $11 y$ altered if the medium is conducting.

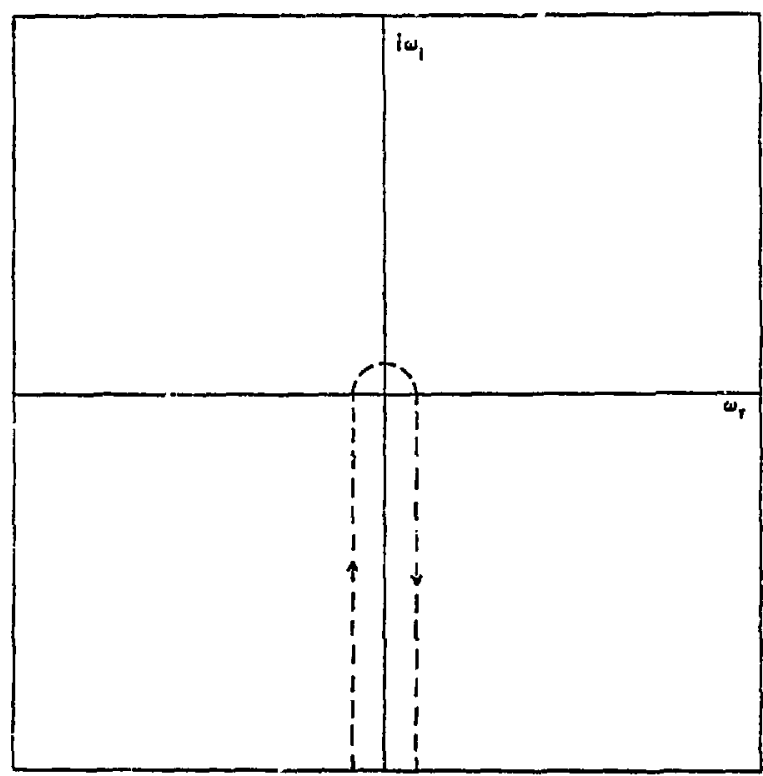

F1g. 1. Integration path used in evaluating the integral of Eq. (12) for positive values of $t$.

For negative yalues of the time $t$, the integial In Eq. (12) must be evaluated by cloging the contour in the positive Imaginary half-plane of $\omega$; because the integrand containg no poles or branch cuts in this region, the result of the integration is zero. Thus, at $r=0$ the vector $\vec{I}_{0}$ jumps discontinuously fror zero for negative times to an inilnite value at $t=0$; for $r>0$ 逪 is always finite and continuous as a function of time and position. The integral of $\vec{I}_{0}$ over all space $1 \mathrm{~s} 4 \pi \mathrm{qcm}$ for any $t$ greater than zero. Frow this it is seen that $\vec{I}_{0}$ starts as a delts function of position ai $t=0$, then decreases in amplitude and spreads out over all space as $t$ 1ncreases. It always retains the character of a delta function in the sents that 1 ts integral over space is constant.

The electric and magnetic fleldg can now be calculated using Eqs. (3) and (4). For this purpose, It $\$ 111$ be assumed that $\vec{m}$ is directed in the tz direction in a Carteilan-coordinate system. For the time being, the actual direction In space will not be specifled, 1.e., the tz direction is not necessar11y upward. The Cartestan components of the electric and magnetic fields are easily calculated from the above equations. If these components are 
comblned to express the flelds in sphertcal coordinates with 0 along the polar axis, the norvanishing components are:

$H_{r}=\frac{16 m q c}{\sqrt{\pi}} \frac{e^{-r^{2} / 4 q c t}}{(4 q c t)^{5 / 2}} \cos \theta$,

$H_{\theta}=\frac{-16 \pi q c}{\sqrt{\pi}}\left(1-\frac{r^{2}}{4 q c t}\right) \frac{e^{-r^{2} / 4 q c t}}{(4 q c t)^{5 / 2}} \sin \theta$,

$E_{\phi}=\frac{80 m q^{2} c}{\sqrt{\pi}}\left(1-\frac{2}{5} \frac{r^{2}}{(4 q c t)}\right) \frac{r e^{-r^{2} / 4 q c t}}{(4 q c t)} \sin \theta$.

From these equations, it is seen that the characteristic time of the fleld varlation is

$$
t_{r}=r^{2} / 4 q c
$$

For $r \approx 300 \mathrm{~m}$ and $q=30 \mathrm{~cm}, t_{r} \approx 0.25 \times 10^{-3} \mathrm{sec}$. Thus, a nuclear explosion at $300 \mathrm{~m}$ underground should produce a VLF signal at the surface of the ground. The olgnal's time dependence is determined by the grou.ad's electrical properties if the source's time dependence is much faster than VLF.

The time dependences of $H_{r}, H_{\theta}$, and $E_{\phi}$ are shown 1n Figs. 2, 3, and 4, respectively.

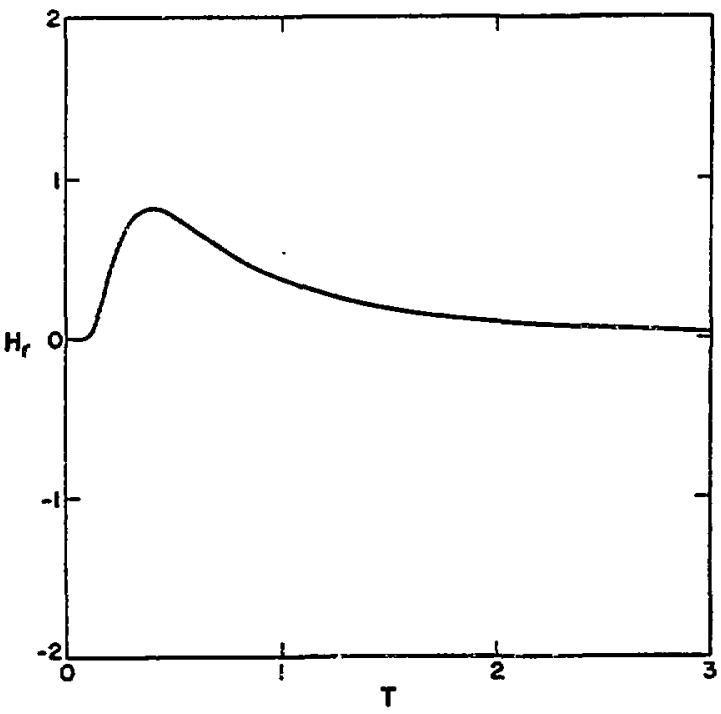

F18. 2. Plot of $T^{-5 / 2} e^{-1 / T}$ where $T=t / t \cdot$ This gives the time dependence of $H_{r}$ ünderground. For unlts, see text.

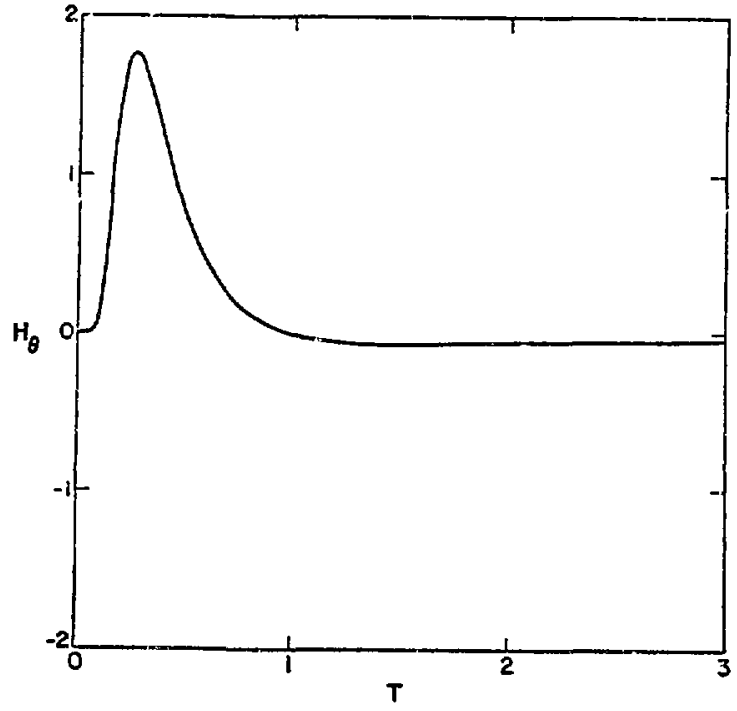

F.g. 3. P1ot of $-T^{-5 / 2}\left(1-T^{-1}\right) e^{-1 / T}$ where $T=t / t$ This gives the time dependence of $\mathrm{H}_{\theta}$ underground. For unt.ts, see the text.

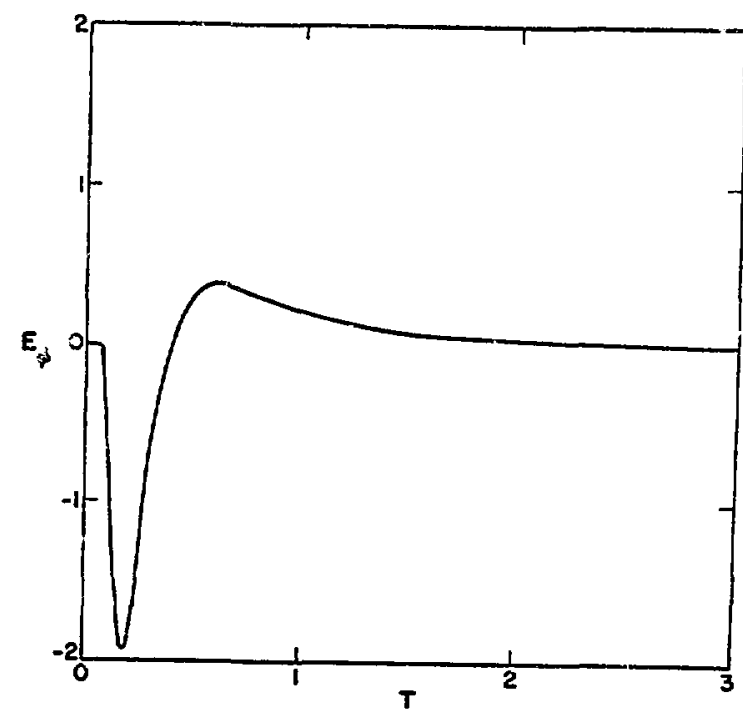

F1g. 4. Plot of $T^{-7 / 2}\left(1-0.4 \mathrm{~T}^{-1}\right) \mathrm{e}^{-1 / \mathrm{T}}$ where $\mathrm{T} \times \mathrm{t} / \mathrm{t}$. ThIs gives the time dependence of $E_{p}$ under ground. For units, see the text.

In these figures, $t$ ime $1 \mathrm{~g}$ exprissed in units of $t_{\text {; }}$. The untts of $\mathrm{H}_{\mathrm{r}}$ and $\mathrm{H}_{\theta}$ are $16 \mathrm{mqc} /\left(\mathrm{r}^{5} \sqrt{\pi}\right)$ multaples: by $\cos \theta$ for $H_{r}$ and $\sin \theta$ for $H_{\theta^{*}}$. For $E_{\phi^{\prime}}$, the units are $80 \mathrm{mg}^{2} \mathrm{c} \sin \theta /(\mathrm{r} \sqrt[6]{\pi})$. Thue, the electric tieid Is smaller than the magnetic field by a factor of 
about $5 q / r$ for $r \gg q$. These figures give the time dependence of the underground signal for any $r$ that is large compared to $q$ in terms of the value of $t_{r}$ approprlate to that $r$. Note, however, that $t_{r}$ is proportional to the ground conductivity, which may differ by as much as an order of magattude from the value assi:med nere.

Tatro ${ }^{3}$ has observed the $2 \mathrm{MP}$ produced at the ground surface near ground zero by an underground nuclear explosion, using magnetometers and 300-ftradius, horizontal, loop antennas. His instrumentation was intended for frequencies in the ELF to dc range; however, the largest amplitude part of the signal he observed was an Initial pulse with a width that appears to be in the millisecond range. Since this experinent was intended to measure signals of linger time duration, the detalls of the recorded pulse cannot be very well determined because of the sliw sweep speed. Moreover, because of the sensors used it probably did not glve a good representation sf the actual signal, particularly for the magnetometers. However, this pulse does seem to have the time dependence of the signals calculated here. Its amplitude was recorded as $n 50$ gammas $(\gamma)$. This initial pulse was followed by a more slowly varying signal of smaller amplitude. Possibly this slowly varylng signal is partly due to the slowly varying talls of the calculated signals in Figs, 2 and 3.

Since the time dependence of the recorded pulses was outside the intended frequency response of the instrumentation, it is probably reasonable to regard $50 \gamma$ as a lower 11 it to the amplitude of the observed signal. With this as:umption, (Eqs. (14) - (16) can be used to extrapoiate the stignal back to the region near the burst point. Both $H_{r}$ and $H_{\theta}$ are functions of the form $\left(1 / r^{5}\right) F\left(t / t_{r}\right)$. The signel was observed at a distance $r=h \simeq 1000 \mathrm{~g}$ from the burst point. If the signal was $50 \gamma$ with mililsecond time dependence at $r=h$, then at $r=h / 10=100 q$ it would be $50 \mathrm{G}$ with t1me dependence in the $10 \mu \mathrm{sec}$ range. The peak energy density in the magnetic field would be abcut $100 \mathrm{ergs} / \mathrm{cm}^{3}$ at this distance. If this energy density occupled a volume equivalent to that of a sphere of $30 \mathrm{~m}$ radius, the total energy would be $\sim 10^{13}$ ergs. For comparison, $1 \mathrm{kt}$ of TNT is equivalent to $4.2 \times 10^{19}$ ergs. Since the peak electric field $1 s \sim 5 q / 2$ times the peak maznetic field, this implies that at $r=h / 10=100 \mathrm{q}$ the electric field $\mathrm{E}_{\phi} \simeq \mathrm{H}_{\theta} / 20 \simeq 2.5$ statvolt $/ \mathrm{cm}=7.5 \times 10^{4} \mathrm{v} / \mathrm{m}$, and if the conductivity is st $11110^{-2}$ mo/m this close to the burst point the peak current density would be $750 \mathrm{~A} / \mathrm{\pi i}^{2}$. As noted above, these values probably represent lower limits to the fleld strengths and current densities consistent with the signal observed at the ground surface. This gives an Indication of the nature of the source that would be required to explain the observed signal.

In Fig. 5 the fleld lines of $H$, whych lie in planes of constant $\phi$, are plotted. Also, in this figure the values in the equatorial plane of $E_{\phi}$ in units of $80 \mathrm{mg}^{2} \mathrm{c} /\left(\sqrt{\pi}(4 q \mathrm{q} t)^{3}\right)$ and $H_{\theta}$ in units of $16 \mathrm{mcc} /\left(\sqrt{\pi}(4 \mathrm{qct})^{5 / 2}\right)$ are plotted vs distance from the burst point. The distances $x$ and $z$ are expressed in units of

$$
a=\sqrt{4 q c t} .
$$



Fig. 5. The magnetic lines of force in the underground regluis. These lines of force (shown as solid 1ines) lie in a plane of constant $\phi$ which in this figure is taken to be the $x-z$ plane. The field has axial symmetry about the polar, or $z$, axis. A.lso shown are the values of $E_{\phi}$ and $H_{\theta}$ in the equatorlal or $x-y$ plane. The scale on the vertical axis gives the value of $z$ as well as of $\mathrm{E}_{\phi}$ and $\mathrm{H}_{\theta}$. For unfts, see the text. 
For $t=10^{-6} \mathrm{sec}, a \simeq 20 \mathrm{~m}$ and for $t=10^{-4} \mathrm{sec}$ $a \simeq 200 \mathrm{~m}$. Thus, the electromagnetic field requires $\sim 10^{-4} \mathrm{sec}$ to diffuse from the burst point to the ground surface. This time delay can also be seen In Figs. 2 - 4.

Figure 5 shows that the electric current density in the ground, $\sigma E_{\phi}$, flows in a ring about the $z$ axis. Thts ring expands ilke a smoke ring and carries its associated magnetic field with it. Because of the high electrical conductivity of the medium, the electric field necessary to drive the current is small comparea to the magnetic field. It was shown above that $E_{\phi}$ is $\sim 5 q / r$ times $H_{\theta}$, so the disturbance becomes largely a magnetic phenomenon at a distance of a few hundred meters from the source. Both $\vec{E}$ and $\vec{J}$ are solenoldal vectors, so there is no electric charge distribucion associated with the disturbance, as must surely be the case for times later than a few microseconds within such a highly conducting medium. A more general underground EMP could consist of a gum of solutions of this type, each with its source uccurring withir, a time span of a few infroseconds and within a volume of dimenstons several meters or less. However, far frcm the sources, such a sum of solutions would look approximately like a single solution of the above type with $\overrightarrow{i n}$ representing the vector sum of all of the source dipoles if this sum did not vanisin. Thus, 1t is reasonable to belleve that the above soiution represents a qualitatively correct plcture of. the underground EMP. The important assumptions on which it is based are that the medium's conductivity is so high that the current must be mainly solenoldal and that the magnetic-dipole part of the source is more important than higher order mult1poles.

Since the magnetic dipole in the source is not necessarily vertical, the ring current assoctated with the mitted fleld will intersect the ground surface, which it cannot fiow through, and therefore be perturbed in some complicated way. Th1s w111 in turn perturb the signal produced at the ground sirface and consequently further complicate the interpretation of measured field strengths in terms of the source strength or orlentation. Clear$1 y$, the only way to obtain quantitative results is to Include the effect of che ground surface in the calculation of the electric and magnetic flelds.

\section{SURFACE EFFECTS AND THE RADIATED SIGNAI}

To satisfy the boundary conditions at the ground surface, one must add to the Hertz vector 就 salculated above a vector that is a solution to the homogeneous form of Eq. (6). Each componet.c of this vector is of the form

$$
\Pi_{1}=\frac{1}{2 \pi} \int_{-\infty}^{\infty} \mathrm{d} \omega \mathrm{e}^{-i \omega t} \mathrm{~F}_{\omega}(\vec{r})
$$

where $F_{\omega}(\vec{r})$ satisfies

$$
\left(\nabla^{2}+\Gamma^{2}\right) F_{\omega}(\vec{r})=0
$$

Below the ground surface, $\Gamma^{2}$ is as defined is Eq. (8) except that $\varepsilon$ is taken to be zero, as already discussed, while above ground $\Gamma=w / c$. Thus, with the ground surface at $z=h$

$$
\Gamma^{2}=\left\{\begin{array}{ll}
(\omega)^{2} / L^{2} & \text { for } z>h \\
1 \omega / a c & \text { for } z<h
\end{array} .\right.
$$

The function $F_{\omega}(\vec{r})$ can be expressed as a sum of functions of the form (for example, see Sommerfeld ${ }^{1}$ )

$$
u(n, \alpha, \omega)=J_{n}(\alpha \rho) \stackrel{+1 n \phi}{\mathrm{e}_{\omega}}(z),
$$

where $\rho, \phi$, and $z$ are cylindrical coordinates with the $z$ axis directed vertically upward, and $\alpha$ and $n$ are real constants. The parameter $n$ must be an integer for this function to be single-valued. $J_{n}(\alpha p)$ is a Bessel function of the first kind and is therefore finite at $p=0$. The function $f_{\omega}(z)$ satisfies

$$
\left(\frac{d^{2}}{d z^{2}}+\Gamma^{2}-\alpha^{2}\right) f_{\omega}(z)=0 .
$$

In the underground region $f_{\omega}(z)$ is thus a linear combination of functions of the form $e^{ \pm 1 \gamma z}$ where

$$
Y=\sqrt{1 \omega / q c-\alpha^{2}}
$$

The sign of the square root is to be chosen such that the real part of $\gamma$ has the same sign as $\omega$ and the Imaginary part is positive for either sign of $\omega$ when both $\omega$ and $\alpha$ are real. 
Above ground, $f(z)$ is of the form

$$
e^{1 \beta z}
$$

where

$$
\beta=\sqrt{\omega^{2} / c^{2}-\alpha^{2}}
$$

Above ground, only the upward-propagating wave is present because the source of radiation is underground. The particular linear combination of upward and downward waves to be used underground is to be determined by the boundary condition that $\vec{\pi}$ must satisfy at the surface.

It is also necessary to express the solution of the inhomogeneous equation $\hbar_{0}$ as a sum of products of Bessel functions and upward- or downward-propagaing waves. This can be done by using the relation

$$
\frac{e^{L \Gamma r}}{r}=1 \int_{0}^{\infty} \alpha d \alpha J_{2}(\alpha \rho) \frac{e^{. L Y|z|}}{\gamma},
$$

which may be derived, for example, by the method used by Sommerfeld (Ref, 1, Pg , 242). The righthand side of this eruation is equivalent to a sum of functlors of the fres given in Eq. (21) with $\mathbf{n}=0$. As expected, this function contains only upward-propagating waves above the source and downsard-propagating waves below 1t. With this resulit, $\overrightarrow{\mathrm{I}}_{0}$, given by Eq. (12), can be written in the region above the source, which is still taken to be at the origin of the coordinate system, and below the ground surface, taken to be the plane $z=h$, as

$\vec{\Pi}_{0}=\frac{\vec{i} \vec{m}}{2 \pi} \int_{-\infty}^{\infty} d \omega e^{-1 \omega t} \int_{0}^{\infty} \alpha d \alpha J_{0}(\alpha \rho) \frac{e^{i \gamma z}}{\gamma}$.

The buundary conditions on $\frac{T}{\pi}$ are determined from those that $\overrightarrow{\mathrm{E}}$ and $\overrightarrow{\mathrm{H}}$ must satisfy; $1 . e .$, the tangentia: comfonents of $\vec{E}$ and $\vec{H}$ must be continuous at $\mathbf{z}=\mathrm{h}$.

First consider the case in which $\vec{m}$ is directed vertically upward, 1.e., has only a $z$ component. Then has only a $z$ component, and from Eqs. (3) (5) the components of $\vec{E}$ are

$$
\begin{aligned}
& E_{x}=-\frac{1}{c} \frac{\partial}{\partial t} \frac{\partial \Pi_{z}}{\partial y}, \\
& E_{y}=+\frac{1}{c} \frac{\partial}{\partial t} \frac{\partial \Pi_{z}}{\partial x},
\end{aligned}
$$

$$
E_{z}=0,
$$

and those of $\vec{H}$ are

$$
\begin{aligned}
& H_{x}=\frac{\partial}{\partial x} \frac{\partial \Pi_{z}}{\partial z}, \\
& H_{y}=\frac{\partial}{\partial y} \frac{\partial \Pi_{z}}{\partial z}, \\
& H_{z}=-\left(\frac{\partial^{2}}{\partial x^{2}}+\frac{\partial^{2}}{\partial y^{2}}\right) \Pi_{z},
\end{aligned}
$$

The continuity of the tangentlal components of $E$ and $H$ implies that both $\pi_{z}$ and $\partial \Pi_{z} / \partial z$ are cont $1 n-$ uous at $z=h$ for this case. Since $\Pi_{z}$ is continuous, it follows that $\mathrm{H}_{2}$ is also continuous. For a vertical magnetic dipole, the electric fleld has no vertical component.

Now consider the case of a horizontal magnetic dipole in the $x$ direction. Such a source will produce a th vector with an $x$ component; however, the boundary conditions at $z=h$ cannot be satisfied unless th also has a vertical component $\Pi_{z}$. This is the same situation that Sommerfeld ${ }^{1}$ found for the case of a horizontal electric or magnetic dipole above an arbitrary earth. If II has $x$ and $z$ components, then from Eqs. (3) - (5) the components of $\vec{E}$ are

$$
\begin{aligned}
& E_{x}=-\frac{1}{c} \frac{\partial}{\partial t} \frac{\partial \Pi_{z}}{\partial y}, \\
& E_{y}=-\frac{1}{c} \frac{\partial}{\partial t}\left(\frac{\partial \Pi_{x}}{\partial z}-\frac{\partial \Pi_{z}}{\partial x}\right), \\
& E_{z}=+\frac{1}{c} \frac{\partial}{\partial t} \frac{\partial \Pi_{x}}{\partial y},
\end{aligned}
$$

and those of $\overrightarrow{\mathrm{H}}$ are

$$
\begin{aligned}
H_{x}= & \frac{\partial}{\partial x} \nabla \cdot \vec{H}-\left(\frac{\varepsilon}{c^{2}} \frac{\partial^{2}}{\partial t^{2}}+\frac{4 \pi \sigma}{c^{2}} \frac{\partial}{\partial t}\right) \Pi_{x} \\
= & \frac{\partial}{\partial x} \frac{\partial \Pi_{z}}{\partial z}-\left(\frac{\partial^{2}}{\partial y^{2}}+\frac{\partial^{2}}{\partial z^{2}}\right) \Pi_{x}, \\
H_{y} & =\frac{\partial}{\partial y} \nabla \cdot \vec{\Pi}, \\
H_{z} & =\frac{\partial}{\partial z} \nabla \cdot \vec{\Pi}-\left(\frac{\varepsilon}{c^{2}} \frac{\partial^{2}}{\partial t^{2}}+\frac{4 \pi \sigma}{c^{2}} \frac{\partial}{\partial t}\right) \Pi_{z} \\
& =\frac{\partial}{\partial z} \frac{\partial \Pi_{x}}{\partial x}-\left(\frac{\partial^{2}}{\partial x^{2}}+\frac{\partial^{2}}{\partial y^{2}}\right) \Pi_{z}
\end{aligned}
$$


The tangential components of E and t are continuous at $z=h$ if each of the following quantities Is continuous there

$$
\frac{\partial \Pi_{x}}{\partial z},\left(\frac{\varepsilon}{c^{2}} \frac{\partial^{2}}{\partial t^{2}}+\frac{4 \pi \sigma}{c^{2}} \frac{\partial}{\partial t}\right) \Pi_{x}, \Pi_{z}, \nabla \cdot \vec{J} .
$$

Note that this implies that $\mathrm{E}_{z}$ is not continuous at $z=h$. The quantity that is continuous there is

$$
\left(\frac{\varepsilon}{c^{2}} \frac{\partial^{2}}{\partial t^{2}}+\frac{4 \pi \sigma}{c^{2}} \frac{\partial}{\partial t}\right) E_{z}=\frac{4 \pi}{c^{2}} \frac{\partial}{\partial t}\left(\frac{\varepsilon}{4 \pi} \frac{\partial E_{z}}{\partial t}+\sigma E_{z}\right)
$$

The quantity in parenthesis on the right-hand side is fust the sum of the displacement current plus the conduction current. From Eq. (2) it follows that the divergence of this quantity is zero. Therefore, Its normal component must be sontinuous across any surface. This is the reason for the complicated boundary conditions that $\vec{I}$ must satisfy when $\vec{m}$ is not vertical and there is therefore a vertical electric-field component. Since $\Pi_{x}$ is not continuous at $z=h$, the divergence of II cannot be continuous unless $\vec{j}$ has a 2 component such that the discontinuity in $\partial \Pi_{z} / \partial_{z}$ cancels that in $\partial \Pi_{x} / \partial_{x} \cdot$ Below ground the dieplacement current is negligible compared to the conduction current, but above ground the conduction current is zero. Therefore, any vertical conduction current below ground must connect nearly continuously to a vertical displacement current above ground. Thus, if the frequency $\omega$ is small compared to $4 \pi \mathrm{J} / \mathrm{t}$, then the amplitude of the vertical electric field fust above the surface is larger than that just below the surface by the factor $4 \pi \sigma / \varepsilon \omega$.

Actually, this mathematical discontinulty is spread through a layer of the earth extending from the surface to a depth of at least several meters because of conductivity variations with iepth near the surface. In this region, the vertical electric field (and possibly also the horizontal field) may be expected to vary rapidly with depth and with local variations in the earth conductivity.

Probably the actual source $\vec{m}$ of the observed underground EMP has both horizontal and vertical components. Because the boundary conditions on differ for these two components, it is necessary to consider the cases of horizontel $\vec{m}$ add vertical $\vec{m}$ separately. Most of the discus:aion will be devoted to the case of a horizontal $\vec{m}$ because it is the most complicated. Also, it turns out that far frcm the source the signal due to a horizontal $\vec{m}$ is much larger than that due to a vertical $\vec{m}$ of the same magn:tude.

For a source $\vec{m}$ in the $x$ direction, the $x$ component of $\vec{I}$ above ground is of the form

$$
\begin{gathered}
\Pi_{x}=\frac{i m}{2 \pi} \int_{-\infty}^{\infty} d \omega e^{-i \omega t} \int_{0}^{\infty} \alpha d \alpha J_{0}(\alpha p) \\
x \frac{b e^{1 \beta(z-h)}}{\gamma} e^{1 \gamma h} \quad z>h,
\end{gathered}
$$

whereas above the source but below the ground surface,

$$
\begin{aligned}
\Pi_{x} & =\frac{i m}{2 \pi} \int_{-\infty}^{\infty} d \omega e^{-1 \omega t} \int_{0}^{\infty} \alpha d \alpha j_{\sigma}(\alpha \rho) \\
& \frac{e^{1 \gamma(z-h)}+a e^{-1 \gamma(z-h)}}{\gamma} e^{1 \gamma h} \quad 0<z<h,
\end{aligned}
$$

where $a$ and $b$ are constants that depend on $\alpha$ and $w$. From the above discussion it is clear that this $\pi_{x}$ is a solution of the homogeneous form of Eg. (6) both above and below ground fo: $z>0$. If a is zero, then $\Pi_{x}$ for $0<z<h$ reduces to $\Pi_{0}$ as expreased in Eq. (26). Underground, the part of $\pi_{x}$ proportional to a represents a wave reflected downward from the surface, whereas above ground $\pi_{x}$ contains only an upward wave that is transmitted through the surface. The factors $e^{1 \gamma h}$ (whlch, 11ke $a$ and $b$, depend on $\alpha$ and $w$ ) were Inserted only to simplify the expressions for $a$ and $b$.

From the boundary conditions found above for ${ }^{I I}$, It is easily shown that $a$ and $b$ satisfy

$$
\beta b=\gamma(1-a) \quad \text {, }
$$

and

$$
\omega b=4 \pi 1 \sigma(1+a)
$$

where again in the underground region $\varepsilon \frac{\omega^{2}}{c^{2}}$ is neglected compared to $\frac{4 \pi i \sigma \omega}{c^{2}}$. From these equaitions $1 t$ follows that

$$
\begin{aligned}
& a=-\frac{\beta+1 \frac{\omega}{c} q \gamma}{\beta-1 \frac{\omega}{c} q \gamma}, \\
& b=\frac{2 \gamma}{\beta-1 \frac{\omega}{c} q Y},
\end{aligned}
$$

which completes the determination of $\Pi_{x}$. 
The determination of $\pi_{z}$ depends on the continutiy of $\nabla \cdot \frac{t}{1}$ which involves $\partial \Pi_{x} / \partial x$. Since $\Pi_{x}$ is Independent of $\phi$, It foilows that

$$
\frac{\partial \Pi_{x}}{\partial x}=\frac{\partial \rho}{\partial x} \frac{\partial \Pi_{x}}{\partial \rho}=\cos \phi \frac{\partial \Pi_{x}}{\partial \rho},
$$

and, since $\partial J_{0}(\alpha \rho) / \partial \rho=-\alpha J_{1}(\alpha \rho)$, the above expresslons for $\Pi_{x}$ can be differentiated with respect to $x$ to cbtain

$$
\begin{aligned}
\frac{\partial \Pi_{x}}{\partial x} & =-\frac{1 m}{2 \pi} \int_{-\infty}^{\infty} d \omega e^{-1 \omega t} \int_{0}^{\infty} \alpha d \alpha J_{1}(\alpha \rho) \cos \phi \\
& x \alpha b \frac{e^{1 \beta(2-h)}}{\gamma} e^{1 \gamma h} \quad z>h,
\end{aligned}
$$

$$
\begin{aligned}
\frac{\partial I}{\partial x} & =-\frac{1 m}{2 \pi} \int_{\infty}^{\infty} d \omega x^{-1 \omega t} \int_{0}^{\infty} \alpha d \alpha J_{1}(\alpha \rho) \cos \phi \\
& x \alpha \frac{e^{I \gamma(z-h)}+a e^{-1 \gamma(z-h)}}{\gamma} e^{1 \gamma h} \quad 0<z<h .
\end{aligned}
$$

From these equations it follows the: $\Pi_{2}$ must contain $J_{1}(\alpha p) \cos \phi$ rather than $J_{0}(\alpha \rho)$. Also, because the source of $\pi_{z}$ is the electric current induced in the surface of the ground, $\pi_{z}$ must contain only an upward-propagating wave for $z>h$ and $a$ downward propagat 1ng wave for $z<h$. Thus, $\Pi_{2}$ can be written as

$$
\begin{aligned}
& \Pi_{z}=\frac{\ln }{2 \pi} \int_{-\infty}^{\infty} d \omega e^{-1 \omega t} \int_{0}^{\infty} \alpha d \alpha J_{1}(\alpha \rho) \cos \phi \\
& x b^{\prime} \frac{e^{1 \beta(z-h)}}{y} e^{1 Y h} \\
& \text { 2>h, }
\end{aligned}
$$

$\pi_{2}=\frac{1 m}{2 \pi} \int_{-\infty}^{\infty} d \omega e^{-1 \omega t} \int_{0}^{\infty} \alpha d \alpha J_{1}(\alpha \rho) \cos \phi$

$$
x b \cdot \frac{e^{-i \gamma(z-h)}}{\gamma} e^{i \gamma h} \quad z<h .
$$

Here the same constant $b^{\prime}$ has been used both above and below the surface because of the boundary condition that $\Pi_{z}$ be continuous at $z=h$. The requirement that $\partial \Pi x / \partial x+\partial \Pi z / \partial z$ be continuous at $z=h$ Implies that

$$
-\alpha b+1 \beta b^{\prime}=-\alpha(1+a)-1 \gamma b^{\prime},
$$

or

$$
b^{\prime}=\frac{-1 \alpha}{B+\gamma}[b-(1+a)] \quad \text {. }
$$

In all of the Integrals that give expressions for components of $\overrightarrow{\hat{l}}$ near or above the ground surface, the factor $e^{1 \gamma h}$ occurs in the integrand. The wain contribution to the integrals comes from those values of $\alpha$ and $\omega$ for which this factor is $\sim 1$. Equation (23) shows that if $\alpha^{2}$ is simall enough to be neglected, the Imaginary part of $y$ is $\sqrt{w /(2 q c)}$; for larger values of $\alpha^{2}$ the imaginary part of $\gamma$ becomes larger. Thus, the frequencles that contribute significantly are those for which

$$
h \sqrt{\omega /(2 q c)}=\frac{i l}{q} \frac{1}{\sqrt{2}} \sqrt{s q / c} \leq 1 .
$$

Since $h / q=10^{3}$, the important frequenctes are those for which

$$
|w q / c| \leq 10^{-6}
$$

The relative importance of the terms $\beta$ and $1(\omega / c)$ qy in the expressions for $a$ and $b$ can now be examined. If $\alpha^{2} \ll|\omega /(q c)|$, then $|q \gamma|=|\sqrt{1 \omega q / c}|$ $\leq 10^{-3}$ for frequenctes that satiafy inequality (42), and except for a very small part of the range of $\alpha$ about the point where $\beta=0,1$,e., where $\alpha=\omega / c$, the term $I(\omega / c)$ qr $I s$ negligible compared to $B$. Suppose, on the other hand, that $\alpha^{2} \geq|\omega /(q c)|$. Then

$$
\alpha^{2} \geq \frac{\omega^{2} / c^{2}}{T \omega q / c \mid} \geq 10^{6} \omega^{2} / c^{2}
$$

for frequencies that satisfy inequality (42). In this case, from Eqg. (23) and (24) it follows that $|\gamma|=\alpha$ and $|\beta| \simeq \alpha$, so again $|\beta| \gg|1(\omega / c) q \gamma|$. The only part of the range of $\alpha$ where the term $1(\omega / c)$ gr is not negliglbly small conpared to $\beta$ is near $\alpha=\omega / c$, and then

$$
\begin{aligned}
& |\beta i=| \sqrt{2 \frac{\omega}{c}} \sqrt{\frac{\omega}{c}-\alpha \mid}, \\
& \left|\frac{\omega}{c} q \gamma\right|=\left|\frac{\omega}{c} \sqrt{1(\alpha q / c}\right| \leq 10^{-3}\left|\frac{\omega}{c}\right| .
\end{aligned}
$$

there again Inequility (42) is assumed to be satisflad, and use ts made of the fact just established that if $\alpha \simeq \omega / c$, then $\alpha^{2} \ll \omega / q c$, so $q \gamma \simeq \sqrt{1 \omega q / c}$. The range of $\alpha$ over which $|\beta|$ is not much larger than $\left|\frac{\omega}{C} q \gamma\right|$ is thus

$$
|\omega / c-\alpha| \leq 10^{-6}|\omega / c|
$$


1.f Inequality (42) is satiofled. Th1s range is too small to be significant, and In the expressions for $a$ and $b / \gamma$ it 18 good approximation to set $q=0$. Th1:s gives

$$
\begin{gathered}
a \simeq-1, \\
b / \gamma \simeq 2 / B,
\end{gathered}
$$

which gives for $\Pi_{x}$ above and below the surface

$$
\begin{aligned}
& \Pi_{x}=\frac{\operatorname{lm}}{\pi} \int_{-\infty}^{\infty} d \omega e^{-1 \omega t} \int_{0}^{\infty} \alpha d \alpha J_{0}(\alpha \rho) \\
& x \frac{e^{1 \beta(2-h)}}{\beta} e^{1 \gamma h} \quad 2>h \text {. } \\
& \Pi_{x}=\frac{1 m}{2 \pi} \int_{-\infty}^{\infty} d \omega e^{-1 \omega t} \int_{0}^{\infty} \alpha d \alpha J_{0}(\alpha \rho) \\
& x \frac{e^{1 \gamma \gamma^{2}}-e^{-1 \gamma(2-2 h)}}{\gamma} \quad 0<z<h .
\end{aligned}
$$

The fact that the integrand of Eq. (44) is infinite at $\alpha=\frac{\omega}{c}$ causes no difficulty because the integral Itself is well behaved there.

If the above approximate values of $a$ and $b / \gamma$ are substituted into Eq. (40) for $b^{\prime}$, the result is

$$
b^{\prime}=\frac{-2 \operatorname{ta} \gamma}{B(B+\gamma)} \text {, }
$$

and if also the relation $\partial J_{0}(\alpha \rho) / \partial x=-\alpha J_{1}(\alpha \rho) \cos \phi$ 1s used, it is essy to show from Eqa. (38) and (39) that in this approximatiun

$$
\begin{aligned}
& \frac{\partial \Pi_{z}}{\partial z}=\frac{\partial}{\partial x} \frac{i m}{\pi} \int_{-\infty}^{\infty} d \omega e^{-1 \omega t} \int_{0}^{\infty} \alpha d \alpha J_{0}(\alpha \rho) \\
& x \frac{e^{i . \beta(z-h)}}{\beta} \frac{(-\beta)}{\beta+\gamma} e^{1 \gamma h} \quad z>h \text {, } \\
& \frac{\partial I_{z}}{\partial z}=\frac{\partial}{\partial x} \frac{i m}{\pi} \int_{-\infty}^{\infty} d_{\omega} e^{-1 \omega t} \int_{0}^{\infty} \alpha d \alpha J_{0}(\alpha \rho) \\
& x \frac{e^{-1 \gamma(z-h)}}{B} \frac{\gamma}{\beta+\gamma} e^{1 \gamma h} \quad 2<h \text {. }
\end{aligned}
$$

From Eqs. (44) - (47) it is easy to see that $\partial \Pi_{x} / \partial z, \Pi_{z}$, and $\nabla \cdot 7$ are all contiuuous at $z=h$ in this approxiation and therefore actafy their respect lve boundary conàtions. $\left(\Pi_{2}\right.$ is obtained from Eqs. (46) and (47) by simply dividing the integrand hy $1 B$ and $-1 \gamma$, respectively). The discontinuity in $\Pi_{x}$ at $z=h$ is such that $\Pi_{x}$ jumps from zero just beneath the surface to a finite value just above $1 t ;$ thus, these expressions for $\Pi_{x}$ and $\Pi_{z}$ satisfy boundary conditions corresponding to Infinite earth conductivity. The actual earth conductivity is so high that these boundary conditions are a good approxtmation, as can be seen fron the above discussion of the relative Importance of the terms $B$ and $1(\omega / c)$ qr in $a$ and $b$.

Below the suriace, $\pi_{x}$ given by Eq. (45) 1s the sum of the field of a magnetic dipole in the $x$ direction located at the origin plus the field of its image in the minus-x direction located at a height $2 h$ above the or 1 in. For this source configiration, both the vertical electric fleld and the horizontal magnetic fleld approach zero as 2 approaches $h$ from below. The other field componenta are doubled as 2 approaches $\mathrm{h}$ from below. This can be seill, for example, by calculating the contributions to the ileld components due to $\pi_{x}$ alone using Eqs. (29) and (30). However, since $\pi_{z}$ in not zero, this image picture does not correctly represent the underground electric and magnetic fields. In particular, it 18 easy to see that the contributions of $\Pi_{2}$ to $\mathrm{H}_{x}$ and $\mathrm{H}_{y}$ do not In general vanish just below the surface.

Because both and have horizontal components at $z=h$, the energy flux tircough the ground surface, given by $\frac{c}{4 \pi} \times \vec{H}$, can differ from zero. If the electric field has a vertical component above ground, there must be a vertical displacement current there, which, as was polnted out above, must connect almost continuously to a vertical conduction current underground. If the underground conductivity is large, a significant vertical current can exist there even if the underground vertical electric fieid is amall. This vertical current ts the source of a horizontal wagatic fleld, which wust be prepers if there is an ungerd energy flux through the surface. The underground vertical current leads to a charge distribution ... the ground surface, which is the suurce of the vertical electric fleld above ground.

The infinite ground conductivity approximation has been made only in the calculation of $a$ and $b$. The Integrals of Eqs. (44) and (46) still depend on 
the co.zductivity through the function $\gamma$. In particular, it was shown that the factor $e^{1 \gamma h}$ limits the frequencles that contribute significantly to the integrals to low values. This factor would also tend to cut off the $\alpha$ integrals for large $\alpha$ if they were not cut of $f$ sooner for other reasons. In was shown above that the dependence of $\gamma$ on $\alpha$ 1s negligible unless $\alpha$ is much greater than $|\omega / c|$, and if this is so, $\beta=1 \alpha$, and the $\alpha$ integrals will be cut off sooner by the factors $e^{1 \beta(z-h)}$ than by $e^{1 \gamma h}$ if $z$ - $h$ is much greater than $h$. Suppose, on the other hand, that $z-h=0$ but $\rho$ is much larger than $h$. Then when $\alpha$ is large compared to $|\omega / c|$, so that $\beta \simeq 1 \alpha$, and $\alpha \rho$ is large compared to 1 , so that $J_{0}(\alpha \rho)$ approaches its asymptotic form,

$$
\frac{\alpha_{\rho} \rho}{\beta}(\alpha \rho)=-\frac{\cos (\alpha \rho-\pi / 4)}{\sqrt{\pi \alpha \rho / 2}}
$$

which oscillates rapidly as a function of $\alpha$ with slowly decreasing asplitude. If $\rho$ is much greater than $h$, this function always varies more rapidly with $\alpha$ than dces $e^{1 \gamma h}$. The larger $p 18$, the more completely the contributions to the integrals in Eqs. (44) and (46) from alternate half-cycles cancel each other, so again the $\alpha$ Integral will cut of before $\alpha$ becomes large enough to contribute significantly to $\gamma$.

It is useful to define a distance $r$ from the point on the ground surface Just abovs the source to the point where the ffeld is evalusied;

$$
r^{i}=\sqrt{(2-n)^{2}+p^{2}} \text {, }
$$

where it is to be understood that $z-h$ is never less than zero. Then if $r^{\prime} \gg h$, che $a$ dependence of $y$ can be Ignored in Eqs. (44) and (46). In these equations,

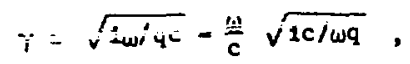

sad from Eq. (42) It follows that for the Important part of the range of $\alpha$ and $\omega,|\gamma| \geq 10^{3}|\omega / c|$. Also, sinca $|\gamma| \gg \mid \alpha j$ for the important part of the range of $\alpha$ and $\omega$, it follows that

$$
|\gamma|>|B| \text {. }
$$

Equations (44) and (46) show that the equations for $\pi_{x}$ and $\Pi_{z}$ abcve ground differ only in that the $\Pi_{z}$ equation contains a derivative with respect to $x$ and the factor $1 /(\beta+\gamma)$ in the tntegrand. Because the $x$ derivative is jroportional to $\alpha$, the $\pi_{z}$ integrand is smaller in absolute value by roughly the factor $|\alpha /(\beta+\gamma)|$, which is much less than 1 . Thus, above ground for $r^{\prime} \gg h, \Pi_{z}$ is negligible compared to $\Pi_{x}$.

In this connection, it is interesting to compare the case in which $\vec{m}$ is in the $z$ direction, rather than in the $x$ direction. Then th has only a $z$ component, which is given by expresstons of the same form as the right-hand sides of Eqs. (31) and (32). However, in this case the boundary conditions on $\pi_{z}$ Imply that

$$
\begin{gathered}
b=1+a, \\
\beta b=\gamma(1-a),
\end{gathered}
$$

or

$$
\begin{aligned}
& a=\frac{\gamma-\beta}{\gamma+\beta}, \\
& \frac{b}{\gamma}=\frac{2}{\gamma+\beta} .
\end{aligned}
$$

Comparing these equations with Eq. (43) shows that because $|\gamma| \gg|\beta|, \Pi_{z}$ due to a vertical source Is very small compared to $\Pi_{X}$ due to a similar hortzontal source for $r^{\prime} \gg h$.

In sumary, the factor $e^{1 \gamma h}$ in the Integrands of the equations for the Hertz vector in the aboveground region limits the contribucing frequenctes to low values. In particular, Eq. (41) is equivaleat to the condition

$$
|\omega / c| \leq q / h^{2} \text {. }
$$

The structure of the integrals giving the components of $t$ above ground far if wit the nource is such that most of the contribution comes from values of $\approx$ and w such that

$$
\alpha^{2} \leq \omega^{2} / c^{2}, \quad B^{2} \leq \omega^{2} / c^{2},
$$

and

$$
\left|\gamma^{2}\right|=|\dot{\omega} ;-=|=\frac{\omega^{2}}{c^{2}}\left|\frac{c}{\omega q}\right| \geq \frac{\omega^{2}}{c^{2}} \frac{h^{2}}{q^{2}} \text {. }
$$


Because $h / q \simeq 10^{3}, \alpha$ and $B$ are negliglbly small compared to $\gamma$ and $(\omega / c)$ q $\gamma$ is negligibly soall compared to $\alpha$ and $\beta$ for the important part of the range of $\alpha$ and $\omega$.

This makes it porsible to use convenient approximations for the quantities be $\mathrm{b}^{i \gamma h}$ and $b^{\prime} \mathrm{e}^{i \gamma h}$ that occur in these integrals when they are evaluated to give the remote aivove-ground signal. These approximations are equivalent to caking the Iimit zero $q$, zero $h$ with $q / h^{2}$ held constant. In this limit, for 2 greater than $h$ the Hertz yector has only a horizontal component whose source is the horizontal component of $\vec{m}$. Th1s is because of the different boundary conditions that $\Pi_{x}$ and $\Pi_{z}$ satisfy, wh1ch, in turn, is due to the fact that only the horizontal component of the source dipole produces a vertica! izintric fleid, which must be much larger just above than just below the ground surface. Consequently, $\pi_{x}$, which gives the vertical electric fleld, must be much larger juat above than just below the surface, whoreas $\Pi_{2}$ is continuous there. The radiated signal for $r^{\prime}$ much greater than $h$ can therefore be obtained from Eqs. (29) and (30) with $\Pi_{2}$ set equal to zero. However, in the underground region $\Pi_{2}$ is not negligible.

To calculate the signal produced at a distance from the source, it remains only to evaluate the integral in Eq. (44). Because the dependence of $\gamma$ on $\alpha$ is not Impirtant, the factor $e^{1 \gamma h}$ can be removed from uncier the integral sign of the $\alpha$ integral. Then this equation becomes

$$
\begin{aligned}
\pi_{x} & =\frac{1 m}{\pi} \int_{-\infty}^{\infty} d \omega e^{-1 \omega t} e^{1 h \sqrt{1 \omega / q c}} \\
& \times \int_{0}^{\infty} \alpha d \alpha J_{0}\{\alpha p) \frac{e^{1 \beta(z-h)}}{\beta} \quad z>h .
\end{aligned}
$$

The a Integral can be evaluated frow Eq. (25). If, In Eq. (25), $\Gamma$ is set equal to $\omega / c$, then $\gamma$, which is $\Gamma^{2}-\alpha^{2}$, becomes $\hat{k}$. This leads to

$$
\frac{e^{I(\omega / c) r^{\prime}}}{I^{T}}=1 \int_{-\infty}^{\infty} \operatorname{col} \alpha J_{0}(\alpha \rho) \frac{e^{I \beta(z-h)}}{B} \text {, }
$$

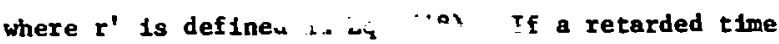

$$
r=t-\frac{r^{\prime}}{c}
$$

ts now Introduced, Eq. (50) can be written as

$$
\Pi_{x}=\frac{m}{\pi} \int_{-\infty}^{\infty} d \omega e^{-1 \omega \tau} \frac{e^{1 h \sqrt{1 \omega /(q c)}}}{r^{\prime}} .
$$

The $w$ integral in this equation is the same as that in Eq. (12), which has already been evaluated. Thus, the resilt for $\Pi_{x}$ above the ground surface and a distance at least several times h from ground zero 18

$$
\begin{aligned}
\Pi_{x} & =\frac{2 h}{r^{\prime}}(4 \pi q c m)(4 \pi q c \tau)^{-3 / 2} e^{-h^{2} /(4 q c \tau)} \\
& =\frac{8 m g c}{\sqrt{\pi} r^{\prime} h} T^{-3 / 2} e^{-1 / 2},
\end{aligned}
$$

where

$$
T=\frac{4 g c T}{h^{2}} \text {. }
$$

Here the characteristic time $t_{h}-h^{2} / 4 q c 18$ a constant, rather than Increasing with $\mathrm{r}^{2}$ as it does underground. Also $\Pi_{x}$ above ground depends on the retarded $t$ Ime $\tau$; it is a hemispherical wave centered on the surface Just above the source. It propagates radially with velocity c while its amplitude decreases as $1 / \mathbf{r}^{\prime}$.

The vertical electric field is given by

$$
E_{z}=\frac{1}{\varepsilon} \frac{\partial}{\partial t} \frac{\partial \pi_{x}}{\partial y}=\frac{1}{c} \frac{\partial}{\partial t} \sin \theta \sin \phi \frac{\partial \pi_{x}}{\partial r^{\top}} \text {, }
$$

where $\theta$ and $\phi$ are the spherical-coordinate angles of $r^{\prime}$ In a coordinate system with 1 ts origin on the surface directly above the source; $\theta$ is measured from the vertical, and $\phi 18$ measured from the direction of $\dot{m}$. From the above expression for $\pi_{x}$, the vertical electric field to

$$
\begin{aligned}
E_{z} & =-\frac{1 \operatorname{smg} c}{\sqrt{\pi} r^{\prime} h^{4}}\left(8 \frac{q^{2}}{h^{2}}\right) \sin \theta \sin \phi \\
& \times\left(\frac{h^{2}}{4 q r^{\top}} \frac{\partial}{\partial T}+\frac{\partial^{2}}{\partial T^{2}}\right) r^{-3 / 2} e^{-i / I} .
\end{aligned}
$$

If $h=300 \mathrm{~m}$, then $t_{h}=h^{2} / 4 q c=0.25 \times 10^{-3}$ sec and $c t_{h}=75 \mathrm{~km}$. When $r^{\prime}$ is small compared to $c t_{h}$, the firat term in the above expression is domInant. This 18 the Induction term; the Induction zone extends is $75 \mathrm{~km}$ from the source. Within the Induction zone, the time dependence of the radiated 
signal depends on distance frum the source. Th1s is shown In F1gs. ó and 7 which give the time dependence of the signal at $r^{\prime}=0.2 \mathrm{ct}_{h}$ and $r^{\prime}=1.6 \mathrm{ct}_{h^{\prime}}$ respectively. The quantity plotted there is

$$
S(T) \equiv \frac{h^{2}}{4 q r^{\prime}} \frac{\partial}{\partial T}+\frac{\partial^{2}}{\partial T^{2}} T^{-3 / 2} e^{-1 / T}
$$

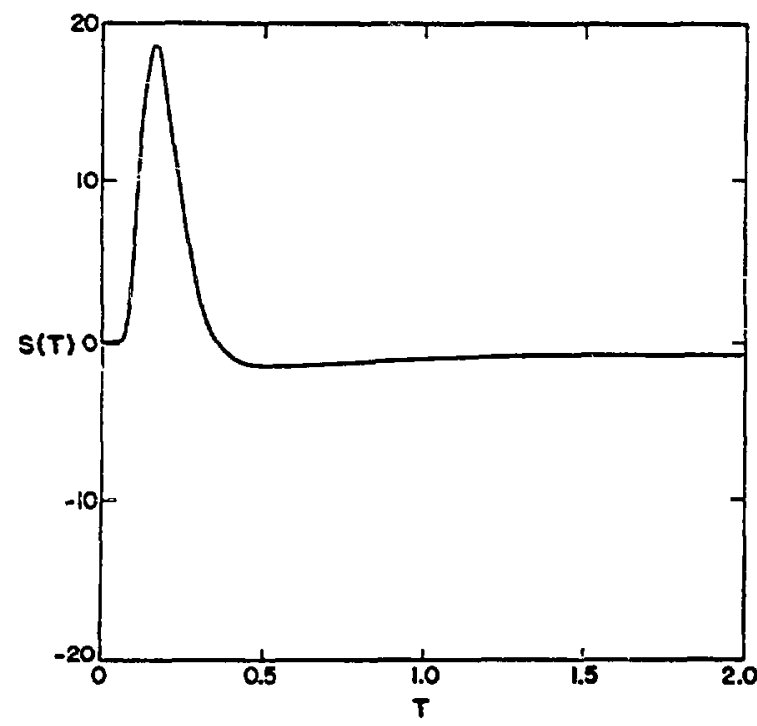

Fig. 6. Time dependence of $E_{z}$ at a distance $r^{\prime}=$ $0.2 c t_{h}$. The time variable $1 \mathrm{~s} T=\tau / t_{h}$. For unfts, see the text.

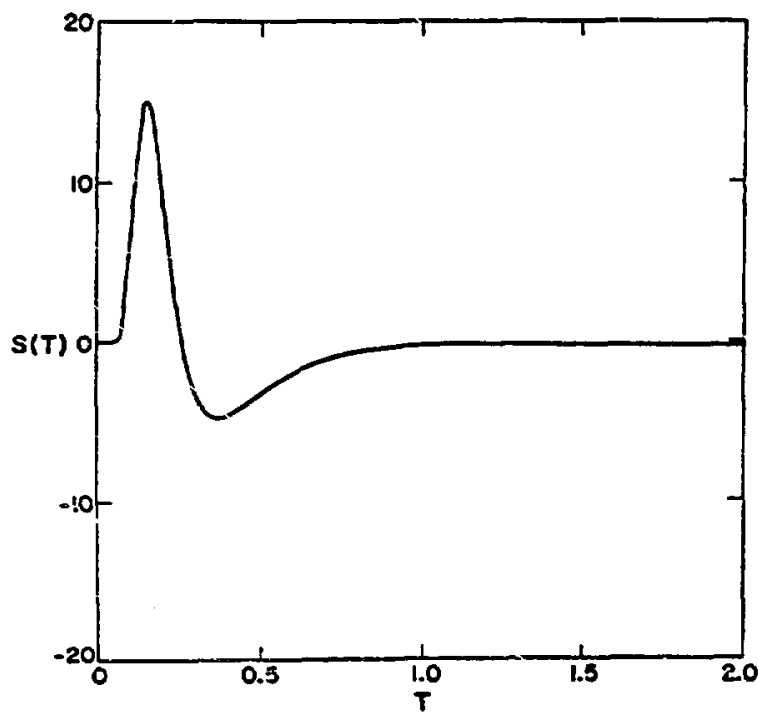

F18. 7. Time dependence of $E_{\text {at a discance }} r^{\prime}=$ $1.6 \mathrm{ct}$. The time variable $1 . T=\tau / t_{h}$. For units, see the text.
If Tatro's measurements, in conjunction with F18. 2 or 3 , are interpreted to mean that

$$
\frac{16 \mathrm{mgc}}{\sqrt{\pi} h^{5}} \simeq 50 \gamma=15 \mathrm{~V} / \mathrm{m},
$$

and $8 q^{2} / h^{2}=8 \times 10^{-6}$, this gives for $E_{z}$, from $E q$. (56),

$$
E_{z}=-(120 \mu \mathrm{V} / \mathrm{m}) \frac{h}{r^{\prime}} \sin \theta \sin \phi S(T) .
$$

Is Indicated, this should probably be interpreted as a lower limis to the expected signal. It was shown that a signal of this amplitude extrapolated back to the time when the peak magnetic fleld was only 30 m from the underground source contalned an energy of $\sim 10^{13}$ ergs, which is less than $10^{-6} \mathrm{kt}$.

Note the dependence of the calculated signal on the sssumed value of the conductivity. If o $1810^{-3}$, Inatend of $10^{-2}$ who/m, q $1 \mathrm{~s}$ increased by a factor of 10 , and the characteristic time and the length of the Induction zone are each reduced by a factor of 10. If Eq. (57) 10 st111 asaumed to hold, the source amplitude $\vec{m} 1 \mathrm{~s}$ reduced by a factor of 10 , and $E_{z}$, given by Eq. (56), 1s increased by a factor of 100 for $r^{\prime} \gg \mathrm{ct}_{h}$.

\section{CONCLUSION}

According to "The Effects of Nuclear Heapona," p. 67,4 an underground nuclear explosion expands the chamber containing the burgt point to a radius of a few tess of meters in a time of a few hundredths of a second. During the explosion and the Inttial part of the expansion, an EMP may be produced which will propagate through the ground faster than the expanalon of the chamber.

An electromagnetic field in the earth causes an electric current, which is itself a source of an electromagnetic field. Thus, shoxtly after, the detonation of a bomb underground, the resulting electromagnet1c fleld witl be contalned in a 20- to 30-mradlus aphere ti: the surrounding eartin, and the induced currents in that sphere may be regarded as the source of the subsequent EMP. Equation (18) showed that the fleld is contained within a radius of $\sim 20 \mathrm{~m}$ af ter about $10^{-6} \mathrm{sec}$, whereas for reasonable values of the electrical conductivity and aielectric constant of the earth, the decay time of an electric charge distribution, $E /(4 \pi \sigma)$, is less 
than $10^{-7} \mathrm{sec}$. It therefore seems reasonable to take the source of the underground EMP to be a sole- 1 notdal current distribution contained in 3 sphere of ح 20- to 30-m radius with a time duration in the usec range. The simplest such source is a magnetic dipole. The events that sccur between the initlation of the explosion and the time when the electroI" gnecic fleld has expanded $t$ " occupy a sphere of this size undoubtedly involve production of electric charge distrtbutions and, therefore, nonsolenotdal current densities, but if the charge distributions decay, the current density must become solenoidal. Also, htgher order multifoles are probably produced, but they can be represented by sums of magnetic $d_{1-}$ poles. Because of the diffuston-like propagation of electromagnetic flelds in a conducting medium, an electromagnetic pulse will be spread in time after propagating some distance underground. Cisnsequently, the detalls of the space and time dependence of 1 ts soirce will be lost. Therefore, it is appropriate to Investigate the EMP produced by an underground point dipole with delta function time dependence.

The underground magnetic-dipole problem was solved in the time domata. The natu:e of the underground signal during the tine before the pulse arrives at the ground surface was determined. This signal consists of a magnetic fleld diffuring through the conducting earth, with a much smaller associated electric field. The electric fleld cauces a current to flow in the earth, which results in Joule hearing with a consequent loss of energy from the electromagnetic disturbance. If the source has delta Eunction time dependence, the signal after propagating $300 \mathrm{~m}$, is a pulse $\sim 10^{-4} \mathrm{sec}$ wide.

The effect of the ground surface was also considered. A careful examination of the conseguences of the boundary onditions at this surface Indicates that the signal due to a horizontal magnetic dipole is much more strongly transmitted through the surface than is that due to a vertical magnetic dipole. The physical reason for this phenomenon is examined in detail. The signal produced by a horizontal magnetic dipole and transmitted through the ground surface to remote points in the atmosphere is calculated. It was shown that if the electromagnetic energy in the diaturbance at a time when it 18 contalned in a 30-mradius sphere is equivalent to $\sim 10^{-6} \mathrm{kt}$, the EMP should be observable above ground out to a f $\mathrm{kw}$ kilometers from ground zero: The time dependence of the remote, aboverground signal depende on the depth of the burst below the surface. For 300-m depth the signal is a pulse in the 0.1 msec range.

\section{REFERENCES}

1. Arnold Somerfeid, Partial Differential Equaciona In Physics (Acadentc Press, Inc., New York, 1949).

2. T. G. Cowling, Mapnetohydrodynamice (Intersclence Publ1shers, Inc., New York, 1957).

3. L. D. Tatre, LOS Alamos Scientific Laboratory, unpub1lshed tata.

4. The Effects of Nuclear Weapons (United States Atomic Energy Commission, 1962). 